\title{
STEREOLOGICAL ESTIMATION OF TUBULAR LENGTH FROM THIN VERTICAL SECTIONS
}

\author{
HELlE V ClaUSEN $^{1,3}$, HANS JG GUNDERSEN ${ }^{2}$ AND LISE G LARSEN $^{3}$ \\ ${ }^{1}$ Department of Obstetrics and Gynecology, Hvidovre University Hospital, ${ }^{2}$ Stereological Research Laboratory, \\ Aarhus University and ${ }^{3}$ Department of Pathology, Næstved Central Hospital, Denmark \\ e-mail: hv.clausen@dadlnet.dk \\ (Accepted August 3, 2000)
}

\begin{abstract}
In this study tubular structures are represented by stem villous arteries of the human placenta. The architecture of the vascular tree in the human placenta makes it appropriate to use vertical histological sections. Describing tubules, estimates of total length and diameter are informative. The aim of the study was to derive a new stereological estimator of the total length of circular tubules observed in thin vertical sections. Design: Dual perfusion fixed human placentas. Systematic, uniformly random sampling of vertical sections. Five- $\mu$ m-sections were stained by haematoxylin and eosin $(\mathrm{H} \& \mathrm{E})$ and the vertical axis was identified in all sections. A test system with cycloid test lines was used. Since tubular surface area is proportional to length and diameter, $S \propto \pi \mathrm{dL}$, surface-weightening is equivalent to diameter $\times$ lengthweightening. As each diameter (extra weight) is known, one may eliminate the diameter-weightening by computing the harmonic mean diameter, which is thus the correct, length-weighted mean tubular diameter, $\bar{d}_{L}={\overline{d_{S}}}^{h}$. Surface area is estimated in the ordinary way from vertical sections, and with unbiased and robust estimates of $\mathrm{S}$ and $\overline{\mathrm{d}}_{\mathrm{L}}$, respectively, total length may be estimated $L=\frac{S}{\pi \bar{d}_{S}{ }^{h}}$. Conclusion: A new
\end{abstract} stereological estimator of total length of a circular tubular structure observed in thin vertical sections is presented.

Keywords: harmonic mean diameter, stem villous arteries, total length, vertical sections.

\section{INTRODUCTION}

In an organ like the human placenta it is very laborious and difficult to quantify differences in structure by morphological methods (Salafia et al., 1992). Semi-quantitative methods may be used, but intraand inter-observer variance make it difficult to draw conclusions from the data. In most morphological studies, due to the size of the organ, conclusions are often based on studies conducted on isolated cotyledons or parts of the placenta. The fine structures of the human placenta has been studied by stereological methods.

In a study performed by Larsen et al. 1995b, we demonstrated a significant reduction in total placental capillary length by approximately $20 \%$ in heavy smokers compared to non-smokers. It is unknown if these changes are expressed in the stem villous arteries as well. The stem villous vessels examined in this study are located in the upper half to two-thirds of the placenta below the chorionic plate. Diameter and length of the arteries are the most interesting parameters. Vertical design was used, because of the anatomy of the stem villous arteries of the human placenta.

Using vertical design a new stereological tool for estimating total length was necessary.

The aims of the study were:

1. to describe the mathematical basis for an estimator of total length of a circular tubular structure from thin vertical sections.

2. to estimate the total length of the stem villous arteries.

\section{MATERIAL}

Thirty fresh placentas, 15 from non-smokers and 15 from heavy smokers, were fixed by dual perfusion, followed by three to four weeks of immersion fixation. A heavy smoker smokes 15 or more cigarettes daily on a regular basis. The weights and volumes of the fixed 
placentas were measured. The reference volume, $\mathrm{V}(\mathrm{ref})$, was measured by immersion in water.

The placental tissues for microscopical stereological analysis were obtained by systematic, uniformly random sampling (SURS) (Gundersen et al., 1981, Kroustrup et al., 1983, Gundersen et al., 1987). The placentas were placed on a quadratic grid and five to nine full-wall blocks were cut perpendicularly to the chorionic plate following the grid lines. From each block of tissue, a vertical full wall section was cut from a random one of the four sides. Finally, 5- $\mu \mathrm{m}$ sections were cut, paraffin embedded and stained with H\&E, for details see Larsen et al., (1995a). The fields of vision were obtained by 'meander'-sampling. Only artery profiles with a circular or ellipsoid shape were included in the sample. Artery profiles with an internal diameter of less than $100 \mu \mathrm{m}$, or a vessel wall that was too thick compared to the diameter of the artery, were excluded (Fig. 1).

\section{METHODS}

\section{MATHEMATICS}

The number of times, $k_{i}$, that the i'th tubule is intersected by IUR lines is proportional to its surface:

$$
\mathrm{k}_{\mathrm{i}} \propto \mathrm{s}_{\mathrm{i}}=\pi \cdot \mathrm{d}_{\mathrm{i}} \cdot \mathrm{l}_{\mathrm{i}}
$$

$s_{i}, d_{i}, l_{i}$ represents the surface, the diameter and the length of the i'th tubule. The simple mean of diameters sampled with IUR lines is surface weighted:

$$
\overline{d_{S}}=\frac{\sum_{i=1}^{m} d_{i}}{m}=\frac{\sum_{j=1}^{n} d_{j} \cdot s_{j}}{\sum_{j=1}^{n} s_{j}}=\frac{n: \overline{d:(l: d)}}{n: \overline{(l: d)}}=\frac{\overline{d:(l: d)}}{\overline{(l: d)}}
$$

where $\Sigma k_{i}=m$ and $n$ is the unknown number of tubules with individual tubule surface area $s_{j}$.

Note that the simple mean of diameters sampled with IUR planes is length weighted

$$
\overline{d_{S}} \neq \overline{d_{L}}=\frac{\overline{d: l}}{\bar{l}}
$$

Comparing the two estimators, we note that $\bar{d}_{S}$ is a diameter weighted version of $\bar{d}_{L}$. The correct estimator is then the harmonic mean of the observations:

$$
{\overline{d_{S}}}^{h}=\frac{1}{\frac{1}{m} \cdot \sum_{i=1}^{m} \frac{1}{d_{i}}}=\frac{\sum_{j=1}^{n} d_{j}: l_{j}}{\sum_{j=1}^{n} \frac{d_{j}: l_{j}}{d_{j}}}=\frac{\overline{d: l}}{\bar{l}}
$$

see e.g. Cruz-Orive, 1980.

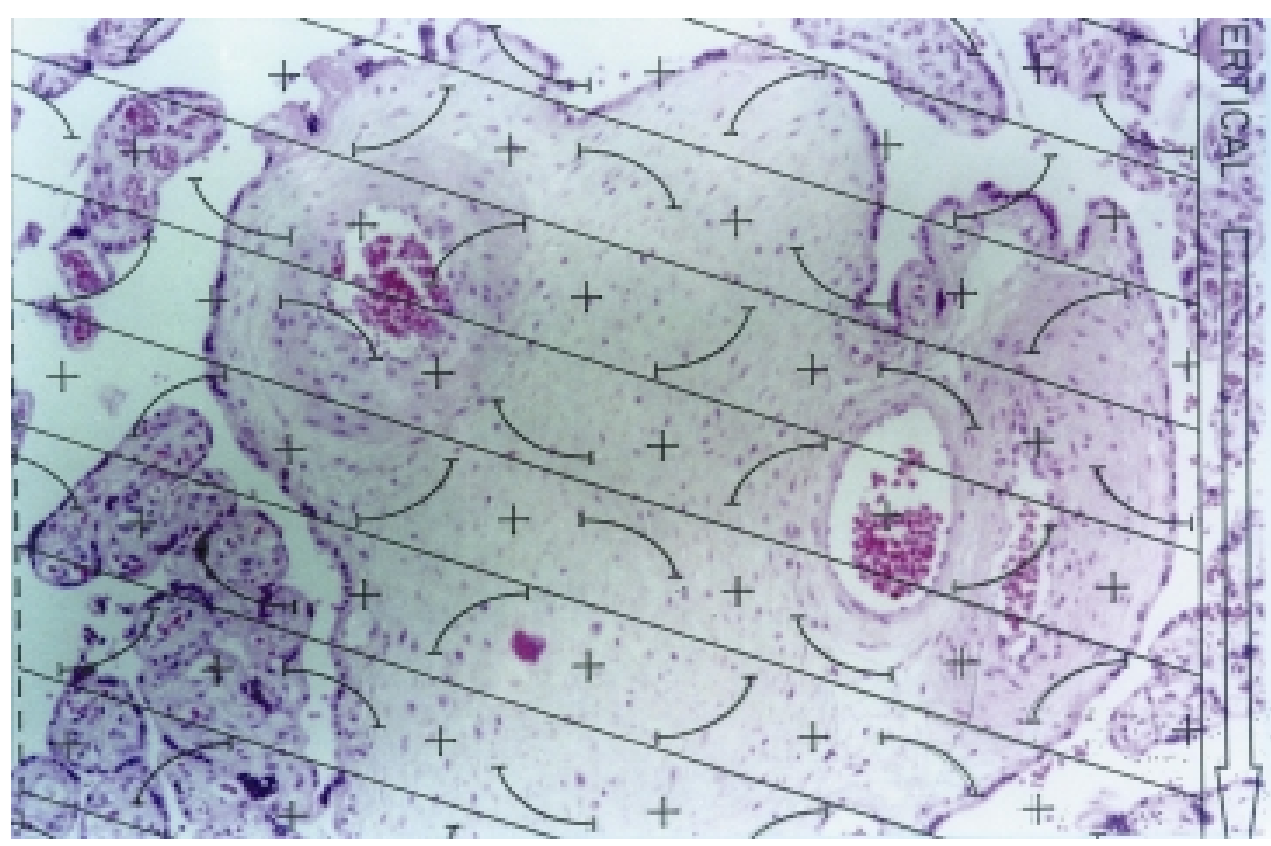

Fig 1. Artery profiles with circular or ellipsoid shape and a diameter more than 100 mikrometer. 


\section{VOLUME, SURFACE AREA, AND LENGTH OF STEM VILLOUS ARTERIES IN THE TOTAL PLACENTA}

All histological sections were blinded with respect to the smoking status of the mother before microscopy and stereological analysis. The vertical sections (Baddeley et al., 1986) were examined in a light microscope with a $10 \times$ objective. A grid of 70 points and 35 cycloids was superimposed on the images on the screen. The area of the sections was estimated by a point grid. In each placenta, estimates of the total volume, surface area, and length of the stem villous arteries with a diameter of 100-300 $\mu \mathrm{m}$ were obtained. When the stem villous artery (ellipsoidal) profile was intersected by a cycloid, the profile diameter was measured. The diameter was measured as the shortest diameter perpendicular to the longest axis of the profile. For a circular tubule, this is the real 3D diameter. The 3D IUR cycloid curves intersect the tubular structure proportional to its the surface area. The measured diameters are thus surface-weighted, $\mathrm{d}_{\mathrm{S}}$, whereas diameters of profiles sampled on ordinary IUR-sections are correctly length-weighted, $\mathrm{d}_{\mathrm{L}}$.

$$
\begin{aligned}
& \text { Volume, } \quad V(\text { sva })=\frac{\Sigma P(s v a)}{\Sigma P(\text { ref })} V(\text { ref }) \\
& \text { Surface area, } \quad S(\text { sva })=\frac{2 \Sigma I(\text { sva })}{\Sigma l} V(\text { ref })
\end{aligned}
$$

$$
\text { Length, } \quad L(s v a)=\frac{S(s v a)}{\pi{\overline{d_{S}}}^{h}}
$$

Where $\mathrm{P}(\mathrm{sva})$ is the sum of points hitting the stem villous arteries, $\mathrm{V}(\mathrm{ref})$ is the placental volume, $\mathrm{P}(\mathrm{ref})$ is the sum of points hitting the reference space. $\Sigma \mathrm{I}$ is the sum of intersections of the cycloids and the structure; $\Sigma l$ is the length of the cycloid multiplied by the number of cycloids in the test frame multiplied by the number of fields.

\section{RESULTS}

The length of the stem villous arteries was estimated from both the endothelial side (lumen harmonic mean diameter) and the external border of the tunica media (the external harmonic mean diameter).

The mean number of artery profiles in the histological sections per placenta was 24.1 for heavy smokers and 28.4 for non-smokers.

\section{DISCUSSION}

The new estimator requires circular cross section in the tubules, as it is in the case for perfusion fixed arteries. An analogous estimator of total length from total volume may be used on arbitrarily oriented sections using diameters of point sampled profiles. Such an estimator would, however, be extraordinarily sensitive to departures from the geometric model of straight circular cylinders without branching.

Table 1. Estimates of total volume, $V$, total surface area, $S$, and total length, $L$, per placenta of the stem villous artery tunica media and endothelium.

\begin{tabular}{lll}
\hline & Heavy Smokers $(\mathrm{n}=15)$ & Non Smokers $(\mathrm{n}=15)$ \\
\hline Placenta volume, $\mathrm{ml}$ & $519(88)$ & $523(117)$ \\
\hline Tunica media & & \\
\hline Volume, $\mathrm{cm}^{3}$ & $1.12(0.66)$ & $1.46(0.45)$ \\
External surface, $\mathrm{cm}^{2}$ & $147(84.7)$ & $200(83.5)$ \\
External diameter, $\mathrm{Ed}_{\mathrm{h}}, \mu \mathrm{m}$ & $212(22.8)$ & $209(29.4)$ \\
Length, $\mathrm{m}$ & $22.0(13.2)$ & $30.8(13.7)$ \\
\hline Endothelium & & $0.034(0.03)$ \\
\hline Volume, $\mathrm{cm}^{3}$ & $0.029(0.02)$ & $149(73.0)$ \\
Surface, $\mathrm{cm}^{2}$ & $120(75.8)$ & $142(19.1)$ \\
Luminal diameter, $\mathrm{Ld}_{\mathrm{h}}, \mu \mathrm{m}$ & $144(14.3)$ & $33.8(16.7)$ \\
Length, $\mathrm{m}$ & $24.0(12.1)$ & \\
\hline
\end{tabular}

mean (SD); there were no significant differences between groups. 
The stem villous vessels examined in this study are located in the upper half to two-thirds of the placenta below the chorionic plate. Making isotropic uniform random (IUR) sections for large structures like stem villous arteries of $100-300 \mu \mathrm{m}$ internal diameter may reduce the possibility of sampling an artery to a point which makes the estimates much smaller. In our study the estimated total volume, surface area and length did not differ significantly when calculated from IUR sections or vertical sections. In this study, L, length of the stem villous arteries was estimated from both the endothelial side (lumen harmonic mean diameter) and from the external border of the tunica media (the external harmonic mean diameter).

The external harmonic mean diameter of the stem villous arteries and the harmonic mean diameter of the lumen did not differ significantly between the two groups (Table 1).

\section{CONCLUSIONS}

1. It is possible to estimate the total length of cylinders from vertical sections using the harmonic mean diameter, ${\overline{d_{s}}}^{h}$ of correctly sampled profiles.

2. The structural dimensions of the stem villous arteries are not affected by cigarette smoking.
A preliminary report of some of the data was presented at the $X^{\text {th }}$ International Congress for Stereology, Melbourne, Australia, 1-4 November 1999.

\section{REFERENCES}

Baddeley AJ, Gundersen HJG, Cruz-Orive LM (1986). Estimation of surface area from vertical sections. J Microsc 142:259-76.

Cruz-Orive L-M (1980). On the estimation of particle number. J Microsc 120:15-27.

Gundersen HJG, Jensen EB (1987). The efficiency of systematic sampling in stereology and its prediction. J Microsc 147:229-63.

Gundersen HJG, Østerby R (1981). Optimizing sampling efficiency of stereological studies in biology: or "Do more less well!" J Microsc 121:65-73.

Kroustrup JP, Gundersen HJG (1983). Sampling problems in an heterogeneous organ: quantitation of relative and total volume of pancreatic islets by light microscopy. J Microsc 132:43-55.

Larsen LG, Clausen HV, Andersen B, Græm N (1995a). A stereologic study of postmature placentas fixed by dual perfusion. Am J Obstet Gynecol 172:500-7.

Larsen LG, Clausen HV, Jønsson L (1995b). Stereological examination of the placenta from women who smoke during pregnancy. Placenta 16:A40.

Salafia CM, Vintzileos AM, Lerer T, Silberman L (1992). Relationships between maternal smoking, placental pathology and fetal growth. J Maternal-Fetal Med 1:90-5. 